\title{
Optimisation of Hand Posture Stimulation Using an Electrode Array and Iterative Learning Control
}

\author{
Timothy A. Exell, Christopher T. Freeman, Katie L. Meadmore, Ann-Marie Hughes, Emma Hallewell and \\ Jane Burridge
}

\begin{abstract}
Nonlinear optimisation-based search algorithms have been developed for the precise stimulation of muscles in the wrist and hand, to enable stroke patients to attain predefined gestures. These have been integrated in a system comprising a 40 element surface electrode array that is placed on the forearm, an electrogoniometer and data glove supplying position data from 16 joint angles, and custom signal generation and switching hardware to route the electrical stimulation to individual array elements. The technology will be integrated in a upper limb rehabilitation system currently undergoing clinical trials to increase their ability to perform functional tasks requiring fine hand and finger movement. Initial performance results from unimpaired subjects show the successful reproduction of six reference hand postures using the system.
\end{abstract}

Keywords-Automated optimisation, functional electrical stimulation, hand movement, surface electrode array.

\section{INTRODUCTION}

$\mathrm{S}_{\mathrm{T}}$ UK, with more than 110,000 first incidents occurring annually [1]. Less than $15 \%$ of patients that experience initial upper-limb paralysis following stroke regain full function [2], which restricts their ability to perform everyday reaching and grasping tasks. A common impairment experienced poststroke is a restriction in hand opening function, caused by a limited ability to activate finger extensor muscles. Functional electrical stimulation (FES) can assist stroke patients in moving their impaired limbs, with clinical evidence [3] supported by neuroscience and motor learning results [4], [5] showing that the therapeutic action of FES is closely associated with the accuracy with which FES supports the patient's movement over repeated attempts at the task. While most clinical FES systems employ open loop or triggered FES control, recent clinical trials have used a biomechanical model of the patient's arm together with model-based controllers. These successful trials confirmed the significant potential of such FES control in providing more effective therapy [6]. Model-based FES control can mitigate physiological changes in the arm, and is critical to

Manuscript received August 31, 2013.

T. A. Exell, C. T. Freeman and K. L. Meadmore are with the School of Electronics and Computer Science, University of Southampton, UK email:(te1@ecs.soton.ac.uk).

A-M. Hughes, E. Hallewell and J. Burridge are with the Faculty of Health Sciences, University of Southampton, UK reduce the effect of noise/disturbance, significantly increase accuracy and enable complex functional tasks to be performed. It is the aim of many upper limb rehabilitation systems in development to transfer use to within the patient's own home. However, no portable or home-based system applying FES to the combined shoulder, arm, wrist and hand has yet been clinically trialled: several stimulate the hand and wrist, including the commercially available Bioness H200 [7], and a very small number of systems apply FES to the arm and shoulder. Implanted Bion electrodes have provided the best overall function, but involve costly surgery. A shortcoming of all surface electrode approaches is lack of selectivity, especially for the hand, due to the complexity of the muscle locations controlling hand movement and the precise positioning of electrodes that this necessitates [8].

The recent emergence of multiple surface electrodes or an electrode array [9]-[12] enables selective stimulation of smaller muscle groups, such as those controlling hand movement [8], [13]. The use of electrode arrays and control hardware to precisely control the site of stimulation also reduces the need to repeatedly reposition surface electrodes. However, due to the close proximity of muscles associated with the hand and wrist, a method of selecting the desired elements for stimulation is required. In addition, due to the small size of stimulation sites for muscles controlling hand movement when compared to other larger muscle groups, intense stimulation signals are required that can lead to discomfort and muscle fatigue [14]. In general the methods of array element selection available in the literature are slow, simplistic and do not exploit an underlying model. The aim of this paper is to develop and verify a quantitative method of finding the optimal stimulation sites of an electrode array for a given movement pattern, providing accurate movement control of the hand and wrist for stroke rehabilitation, whilst minimising set-up time and the need to reposition electrodes. The approach taken utilises iterative learning control (ILC), a control technique that was developed for industrial systems that repetitively complete the same movement. Over repeated performances, ILC continually updates the FES input signals to reduce error in the movement. Recent work has successfully used ILC to regulate the timing and intensity of FES applied to stroke patient's muscles whilst performing planar [15], [16] and 3D [6], [17]-[20] reaching and grasping tasks. In previous clinical trials ILC allowed the required assistive stimulation to be applied to a patient's muscles, while also encouraging voluntary effort by reducing stimulation if a task is performed well. The inclusion of voluntary effort during stimulated movement is an important 
factor for maximising motor recovery during FES therapy [4].

In this paper ILC is used in combination with simple identification procedures replacing the need for an explicit model of the wrist and hand. Once array stimulation patterns are identified for explicit hand gestures, it is shown how these may be incorporated into existing ILC algorithms which control FES applied to the shoulder and arm [6].

\section{METHODS}

A data glove (5DT 14 Ultra, 5DT, USA) was used to collect joint angle data from the fingers. The glove incorporates 14 fiber-optic bend sensors that are positioned over the metacarpophalangeal and proximal interphalangeal joints to measure finger flexion and between each finger to measure abduction. A twin axis electrogoniometer (Model SG75, Biometrics, UK) was used to collect angle data for the wrist joint (flexion/extension and radial/ulnar deviation). A 40 element electrode array (Tecnalia, Spain), comprising $5 \mathrm{x}$ 8 elements, was used to apply the FES. Each of the 40 array elements can be routed to one of four FES channels. The electrode array, data glove and electrogoniometer can be seen in Fig. 1.

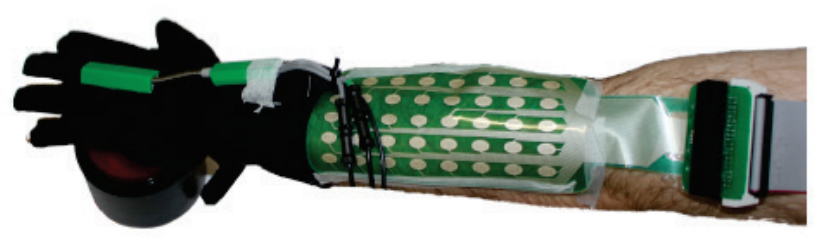

Figure 1: Electrode array with data glove and electrogoniometer used for stimulating and tracking hand and wrist movement.

The array was positioned on each participant's arm as shown in Fig. 1 covering extensor muscles of the wrist and fingers including: extensor carpi radialis longus, extensor carpi radialis brevis, extensor digitorum, extensor pollicis longus, extensor pollicis brevis, extensor indius, and flexor digitorum profundus. Routing of the array elements to the FES channels is performed by custom made RS232 controlled multiplexor hardware, comprising an Arduino board and shift register array (Fig. 2).

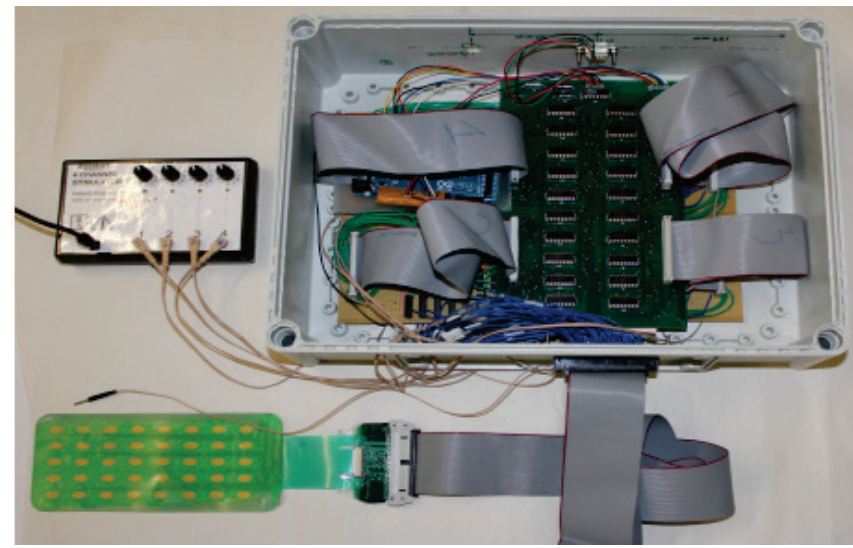

Figure 2: Electrodes array (bottom) with multiplexor hardware and four channel stimulator (top)
A modified commercial stimulator (Odstock, UK) is used to produce the FES signals, where pulsewidth, amplitude and frequency have been selected to produce a smooth muscle contraction [21]. For the stimulation signal, the system produces a $5 \mathrm{~V} 40 \mathrm{~Hz}$ square pulse train with variable pulsewidth for each channel. These pulses are then amplified by the stimulator, resulting in bi-phasic voltage-amplified stimulation. The pulse amplitude for each of the four channels is determined manually, whilst pulsewidth is the control system input and, therefore, is updated automatically. For safety, the stimulation signal pulsewidth is limited to between 0 to $300 \mathrm{~s}$. The system is shown in Fig. 2. At the beginning of each testing session, the stimulator amplitudes are set by routing one channel to two elements of the array. The stimulator is set to output a $300 \mathrm{~s}$ signal whilst the amplitude is slowly increased until a maximum comfortable level is reached. The amplitudes of the remaining four channels are then set to identical levels.

\section{A. Optimisation Procedure}

Preliminary tests with the electrode array have indicated that single element stimulation can induce movement of individual fingers. However, when stimulating just one element of the array, a higher pulsewidth is required to produce movement of the hand and wrist than for traditional larger electrodes. The higher level of stimulation required when stimulating individual elements can cause sensations of discomfort to the participant, reflecting the findings of previous studies into electrode size [22]. Since lower pulsewidths are required to produce movement when stimulating multiple electrode elements simultaneously, hence reducing sensations of discomfort, a stimulation procedure was developed that can stimulate individual elements as well as "blocks" of multiple adjacent elements.

Accordingly, let $u(t)$ be a $m \times 1$ vector of signals which specifies the FES stimulation input signal applied to any set of $m$ single or multiple electrode elements at time $t$. Let $y(t)$ be the $p \quad x \quad 1$ vector of joint angles. The dynamic relationship linking these variables can be written as $y(t)=g(u(t), t)$ where $g(\cdot)$ is a vector function. An explicit algebraic representation of $g(\cdot)$ appears in [23]. The problem of finding a stimulation profile, $u(t)$, which produces the required posture, $y_{d}(t)$, can be expressed as

$$
\text { minimize } f(u(t)), \quad f(u(t))=\left\|y_{d}(t)-g(u(t), t)\right\|^{2}
$$

subject to constraints on $u(t)$. Here $\|\cdot\|$ denotes a suitable signal norm.

This is readily tackled by the ILC framework, however the underlying model $g(\cdot)$ is generally required to be known. As detailed in [23], if only a final posture is required, with the joint trajectories taken to attain it not specified, the problem becomes significantly easier to tackle, and an explicit model of the system is not necessary. Instead an iterative procedure can be taken and involves the following steps:

1) Set $k=0$ and choose an initial input $u_{k}$ to be applied to the set of $m$ electrode elements (or blocks of elements). Note that since only the 'steady-state' problem is considered, the time variable is omitted. 
2) Gradually apply $u_{k}$ to the system experimentally and record the resulting joint angles $y_{k}$ once the hand and wrist has come to rest at its final position. Calculate the postural joint error $e_{k}=y_{d}-y_{k}$

3) Linearize the system about the operating point input $u_{k}$. This is achieved by continuing to apply $u_{k}$, but slowly varying each element of the vector in turn, and recording its individual effect on all the output joint angles. These data are used to produce a pm matrix which is denoted $g^{\prime}\left(u_{k}\right)$.

4) In simulation, solve the problem

$$
\text { minimize } f_{k}(v), \quad f_{k}(v)=\left\|e_{k}-g^{\prime}\left(u_{k}\right) v\right\|^{2}
$$

subject to constraints which are inherited from $u_{k+1}$ via the update in step (5). There are many methods available to solve this problem, such as that described in [23].

5) Calculate the new input

$$
u_{k+1}=u_{k}+v_{k}^{*}
$$

where $v_{k}$ is the solution to the problem in step (4).

\section{6) Increment $\mathrm{k}$ and go to step (2).}

The above procedure is an implementation of Newton method based ILC [24]. The low dimensionality made possible by only specifying the final posture attained (rather than at all times between the initial and final postures) makes it straightforward to include the identification step (3) in place of a predefined system model $g(\cdot)$. Note that:

- Choosing a set of $m$ blocks of array elements that are known to correspond to relevant muscle groups, or to have appeared in previous solutions, reduces the dimension of the problem, and hence the time taken for identification.

- The procedure may be stopped after a single or limited number of iterations in accordance with practical demands-

- Newton method is just one technique in the class of gradient algorithms, and has well defined convergence criteria (see, for example, [25]). It has the benefit of having full theoretical underpinning within unconstrained and constrained ILC [24] approaches. However any approach that iteratively solves an input constrained nonlinear equation while embedding experimental data may be used in its place.

- The above approach results in a stimulation pattern, $u^{*}$, that associates each of the $m$ electrode elements (or blocks of elements) with one of five levels of stimulation (four non-zero levels, plus zero). This pattern of stimulation can be used directly during rehabilitation in an openloop manner, however the accuracy of the movement is likely to degrade due to muscle fatigue, spasticity or other physiological effects.
- To address this problem, the solution can be combined with feedback, where, for example a scalar gain $\gamma$ is applied to $u^{*}$ whose value is proportional with the current overall joint error, $\left\|y_{d}(t)-e(t)\right\|^{2}$.

- Suppose it is desired to incorporate hand tracking within an ILC scheme where other muscles are simultaneously stimulated, and a reference is given for each joint over the entire trial length. Ordinarily this would require a full model of the hand and wrist, however by only allowing hand and wrist muscles to be stimulated by a scalar multiple of $u^{*}$, the dimensionality of the problem is reduced such that a model-free approach (e.g. socalled 'phase-lead ILC' used in [6], [15]) is feasible. Moreover, this approach generalises to using any set of previously identified postures.

\section{B. Verification}

Three different reference postures were selected to verify the optimisation procedure; "pointing" with the index finger, "pinching" between thumb and index finger and an "open" hand posture. These postures incorporated specific finger movements as well as extension of the fingers and wrist, which comprise difficult movements for stroke patients to perform. Examples of the three hand postures are shown in Fig. 3. The iterative optimisation procedure was undertaken on two unimpaired participants who each provided no voluntary effort. The optimised solution was tested for the first 3 trials. Mean normalised error was calculated across all joints for each posture using $100 \mathrm{x}\|e\| \mathrm{x}\left\|r-y_{0}\right\|$, where $y_{0}$ is the starting posture. Resulting error values were expressed as a percentage, with 0 indicating perfect reproduction of the reference posture and 100 indicating no improvement from the starting position.

\section{EXPERIMENTAL RESULTS}

Mean error results for the first 3 optimisation iterations are shown in Table I.

TABLE I. MEAN NORMALIZED ERROR (\%) ACROSS ALL JOINTS FOR ITERATIONS 1-3.

\begin{tabular}{|l|l|l|l|l|}
\cline { 3 - 5 } \multicolumn{2}{c|}{} & Pointing & Pinching & Open \\
\hline \multirow{4}{*}{ Iteration 1 } & P1 & 29.72 & 24.49 & 19.09 \\
& P2 & 28.66 & 35.31 & 22.16 \\
\hline & & & & \\
\hline & & & & \\
& & & & \\
Iteration 2 & P2 & 11.19 & 12.62 & 14.36 \\
& & & 14.98 & 13.02 \\
& & & & \\
\hline
\end{tabular}

Results indicate that each iteration reduced mean error by approximately $30 \%$, with mean error after 3 trials typically being $<5$.

Figure 3 shows the optimal stimulation sites identified from Participant 1, for the pointing, pinch and open hand postures. Current work is underway to extend these 
verification tests to include stroke patients that are unable to extend their hand and fingers voluntarily without assistance.

\section{DISCUSSION}

The aim of this paper was to develop and verify a quantitative method of finding the optimal stimulation sites of an electrode array for a given movement pattern. The optimisation procedure that has been developed can iteratively evaluated the accuracy of hand posture for each available combination of array elements in an automated approach. The presented method incorporates ILC, which has been shown to be a robust method of optimisation calculation [24].
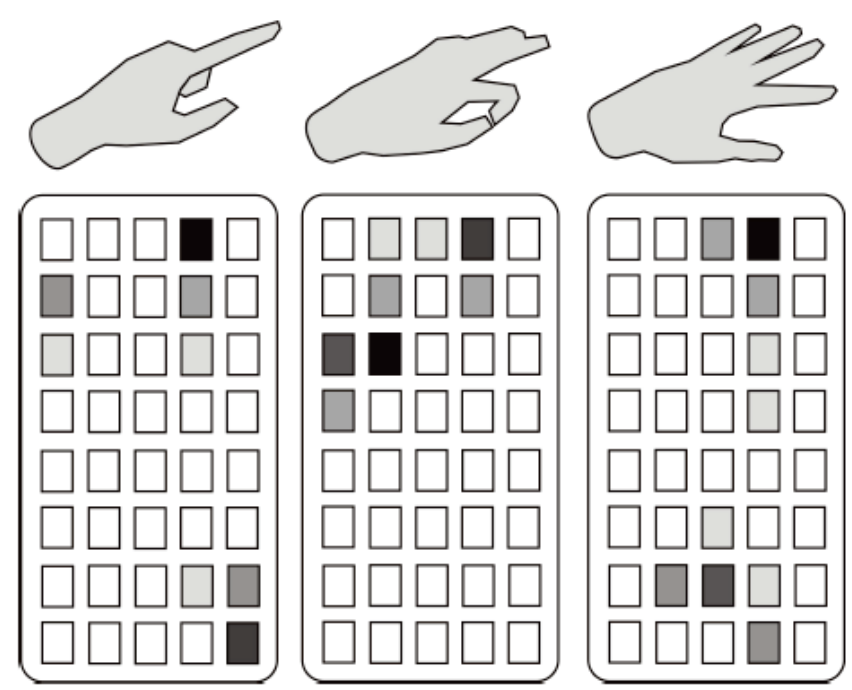

Figure 3: Example of optimal stimulation sites for pointing, pinching and open hand gestures (shading indicates the four non-zero stimulation points)

The procedure was successful in selecting the most appropriate combination of stimulation sites and intensity to produce the desired movement for each participant. Mean joint error was greatly reduced when stimulation was applied to the optimal sites, compared with the starting posture of the hand. The automated selection of array element stimulation has two key benefits. First, the amount of setup time prior to FES therapy can be reduced and the undesirable need to reposition electrodes eliminated [8] and second, the movement produced by FES is improved by the automated selection of the optimal array elements for the desired movement. From the methods developed in this paper, it is possible to combine the use of the electrode array in functional rehabilitation systems incorporating reaching to manipulate objects that currently employ ILC [19].

\section{CONCLUSION}

A system has been developed for the stimulation of forearm muscles using a surface electrode array. The system incorporates an optimisation procedure that automates the selection of array elements that produce the most accurate match to a desired hand posture. The optimisation procedure and stimulation using the identified array elements has been verified experimentally. Results indicate that the system will be useful in FES therapy for training fine grasping and manipulation movements. Collection of data is ongoing and a current feasibility trial is underway using the system with chronic stroke patients for assistance with extension movements of the hand and wrist.

\section{ACKNOWLEDGEMENT}

This work is supported by the Engineering and Physical Sciences Research Council Grant No. EP/I01909X/1, and Bridging the Gap

\section{REFERENCES}

[1] D. of Health., Progress in Improving Stroke Care. National Audit Office, 2010

[2] H. T. Hendricks, J. van Limbeek, A. C. Geurts, and M. J. Zwarts, "Motor recovery after stroke: A systematic review of the literature," Archives of Physical Medicine and Rehabilitation, vol. 83, no. 11, pp. $1629-1637,2002$.

[3] J. R. D. Kroon, J. H. van der Lee, M. J. Ijzerman, and G. J. Lankhorst, "Therapeutic electrical stimulation to improve motor control and functional abilities of the upper extremity after stroke: a systematic review," Clinical Rehabilitation, vol. 16, pp. 350-360, 2002.

[4] J. H. Burridge and M. Ladouceur, "Clinical and therapeutic applications of neuromuscular stimulation: A review of current use and speculation into future developments," Neuromodulation, vol. 4, no. 4, pp. 147-154, 2001.

[5] D. N. Rushton, "Functional electrical stimulation and rehabilitation an hypothesis," Medical Engineering and Physics, vol. 25, no. 1, pp. $75-78,2003$.

[6] C. T. Freeman, E. Rogers, A.-M. Hughes, J. H. Burridge, and K. L. Meadmore, "Electrical Stimulation and Robotic-Assisted Upper Limb Stroke Rehabilitation," IEEE Control Systems Magazine, vol. 32, no. 1, pp. 18-43, 2012.

[7] G. J. Snoek, M. J. IJzerman, F. A. in 't Groen, T. S. Stoffers, and G Zilvold, "Use of the NESS handmaster to restore handfunction in tetraplegia: clinical experiences in ten patients," Spinal Cord, vol. 38, no. 4, pp. 244-249, Apr 2000.

[8] S. O'Dwyer, D. O'Keeffe, S. Coote, and G. Lyons, "An electrode configuration technique using an electrode matrix arrangement for FES-based upper arm rehabilitation systems," Medical Engineering \& Physics, vol. 28, no. 2, pp. $166-176,2006$.

[9] T. Keller, M. Lawrence, A. Kuhn, and M. Morari, "New multichannel transcutaneous electrical stimulation technology for rehabilitation," Conf Proc IEEE Eng Med Biol Soc, vol. 1, pp. 194 197, 2006.

[10] A. Popović-Bijelić, G. Bijelić, N. Jorgovanović, D. Bojanić, M. B. Popović, and D. B. Popović, "Multi-field surface electrode for selective electrical stimulation," Artif Organs, vol. 29, no. 6, pp. 448-452, Jun 2005.

[11] D. B. Popović and M. B. Popović, "Automatic determination of the optimal shape of a surface electrode: Selective stimulation," Journal of Neuroscience Methods, vol. 178, no. 1, pp. 174 - 181, 2009.

[12] A. J. Westerveld, A. C. Schouten, P. H. Veltink, and H. van der Kooij, "Selectivity and resolution of surface electrical stimulation for grasp and release," IEEE Trans Neural Syst Rehabil Eng, vol. 20, no. 1, pp 94-101, Jan 2012.

[13] O. Schill, R. Rupp, C. Pylatiuk, S. Schulz, and M. Reischl, "Automatic adaptation of a self-adhesive multi-electrode array for active wrist joint stabilization in tetraplegic sci individuals," in Science and Technology for Humanity (TIC-STH), 2009 IEEE Toronto International Conference, 2009, pp. 708 -713.

[14] G. M. Lyons, G. E. Lane, M. Clarke-Maloney, J. V. O’Brien, and P. A. Grace, "An investigation of the effect of electrode size and electrode location on comfort during stimulation of the gastrocnemius muscle," Medical Engineering and Physics, vol. 26, no. 10 , pp. 873-878, 2004.

[15] C. T. Freeman, A. M. Hughes, J. H. Burridge, P. H. Chappell, P. L. Lewin, and E. Rogers, "Iterative learning control of FES applied to the upper extremity for rehabilitation," Control Engineering Practice, vol. 17, no. 3, pp. 368-381, 2009.

[16] A. M. Hughes, C. T. Freeman, J. H. Burridge, P. H. Chappell, P. Lewin, and E. Rogers, "Feasibility of iterative learning control mediated by functional electrical stimulation for reaching after stroke," Journal of Neurorehabilitation and Neural Repair, vol. 23, no. 6, pp. 559-568, 2009.

[17] K. L. Meadmore, A. M. Hughes, C. T. Freeman, Z. Cai, D. Tong, J. H. Burridge, and E. Rogers, "Functional electrical stimulation 
mediated by iterative learning control and 3D robotics reduces motor impairment in chronic stroke," Journal of NeuroEngineering and Rehabilitation, vol. 9, no. 32, June 2012.

[18] Z. Cai, D. Tong, K. L. Meadmore, C. T. Freeman, A. M. Hughes, E. Rogers, and J. H. Burridge, "Design \& control of a 3d stroke rehabilitation platform." IEEE Int Conf Rehabil Robot, pp. 1-6, 2011.

[19] C. T. Freeman, T. Exell, K. Meadmore, E. Hallewell, A.-M. Hughes, and J. Burridge, "Computational models of upper limb movement during functional reaching tasks for application in electrical stimulation based stroke rehabilitation," in $4^{\text {th }}$ European Conference on Technically Assisted Rehabilitation, 2013.

[20] T. Exell, C. T. Freeman, K. L. Meadmore, M. Kutlu, E. Hallewell, A. M. Hughes, J. H. Burridge, and E. Rogers, "Goal orientated stroke rehabilitation utilising electrical stimulation, iterative learning and microsoft kinect," IEEE Int Conf Rehabil Robot, pp. 1-6, 2013.
[21] J. R. D. Kroon, M. J. IJzerman, J. J. Chae, G. J. Lankhorst, and G. Zilvold, "Relation between stimulation characteristics and clinical outcome in studies using electrical stimulation to improve motor control of the upper extremity in stroke," Journal of Rehabilitation Medicine, vol. 37, no. 2, pp. 65-74, 2005.

[22] R. Patterson and J. Lockwood, "The current requirements and the pain response for various sizes of surface stimulation electrodes," in Engineering in Medicine and Biology Society, 1991. Vol.13: 1991. Proceedings of the Annual International Conference of the IEEE, 1991, pp. 1809-1810.

[23] A. I. Soska, C. T. Freeman, T. Exell, and E. Rogers, "Surface electrode array based control of the wrist and hand," in IFAC International Workshop on Adaptation and Learning in Control and Signal Processing, 2013, pp. 164-169.

[24] C. T. Freeman and Y. Tan, "Iterative learning control with mixed constraints for point-to-point tracking," IEEE Transactions on Control System Technology, vol. 21, no. 3, pp. 604-616, 2012.

[25] J. M. Ortega and W. C. Rheinboldt, Iterative Solution of Nonlinear Equations in Several Variables. Academic Press, New York, 1970. 